\title{
Membranas de fibra oca de polietersulfona: efeito do líquido interno e da adição de argila
}

\author{
Polyethersulfone hollow fiber membranes: \\ effect of the internal liquid and the \\ addition of clay
}

\author{
Rodholfo da Silva Barbosa Ferreira ${ }^{1}$, Sandriely Sonaly Lima Oliveira ${ }^{1}$, \\ Amanda Melissa Damião Leite ${ }^{2}$, Edcleide Maria Araújo ${ }^{1}$, \\ Hélio de Lucena Lira ${ }^{1}$, Keila Machado de Medeiros ${ }^{1}$
}

\author{
${ }^{1}$ Unidade Acadêmica de Engenharia de Materiais - UAEMa/CCT/UFCG - CEP: 58.429-900, Campina Grande, Paraíba, \\ Brasil. \\ ${ }^{2}$ Escola de Ciências e Tecnologia (EC\&T)/UFRN, CEP: 59078-970, Natal, Rio Grande do Norte, Brasil. \\ e-mail: rodholfoferreira@gmail.com, sandriely_sonaly@hotmail.com, edcleide.araujo@ufcg.edu.br, \\ hélio.lira@ufcg.edu.br, keilamachadodemedeiros@gmail.com amanda.leite@ect.ufrn.br
}

\begin{abstract}
RESUMO
As membranas poliméricas vêm sendo bastante utilizadas no processo de tratamento de efluentes em todo mundo, solucionando problemas médicos, sociais e ambientais. Sendo assim, membranas de fibra oca foram produzidas a partir da polietersulfona (PES), adicionando a argila Cloisite $\mathrm{Na}-$ CLNa e também inserindo o solvente N,N dimetilformamida (DMF), com o intuito de modificar a morfologia da membrana. A argila foi caracterizada por fluorescência de raios-X (FRX) e difração de raios-X (DRX). Por FRX, ficou visto que a argila apresentou característica naturalmente sódica e com pouco materiais acessórios. O DRX mostrou que a Cloisite $\mathrm{Na}$ possui característica de uma argila montmorilonita contendo íons $\mathrm{Na}^{+}$. As membranas foram caracterizadas por ângulo de contato (membrana plana), microscopia eletrônica de varredura (MEV) e medidas de fluxo. Por meio das medidas do ângulo de contato foi visto que a presença de argila favoreceu a um aumento da hidrofilicidade das membranas. Nas membranas de fibra oca, foi observado por MEV que a presença da argila modificou a morfologia da membrana, alterando a uniformidade dos "fingers" e dos macroporos e modificando a camada densa no centro do suporte poroso, A adição do ácido no líquido interno modificou ainda mais a morfologia, diminuindo a camada densa no centro do suporte poroso da membrana e também alterando os "fingers" e os macroporos, tendo uma maior uniformidade dos mesmos em relação a membrana sem a presença do DMF no líquido interno. A partir das medidas de fluxo foi possível observar que a membrana contendo 5\% de CLNa com a presença do solvente no líquido interno obteve um maior fluxo com o tempo, isto ocorreu devido à morfologia mais uniforme apresentada da membrana.
\end{abstract}

Palavras-chave: polietersulfona, membranas, fibra oca, medidas de fluxo.

\section{ABSTRACT}

Polymer membranes have been widely used in the treatment of effluents worldwide, solving medical, social and environmental problems. Thus, hollow fiber membranes were produced from polyethersulfone (PES) with adding Cloisite $\mathrm{Na}$ - CLNa clay and also by inserting the $N, N$ - dimethylformamide (DMF) solvent, in order to modify the membrane morphology. Clay was characterized by X-ray fluorescence (FRX) and X-ray diffraction (XRD). By FRX, it was seen that the clay presented naturally sodium and with little accessory materials. The XRD showed that Cloisite $\mathrm{Na}$ has a characteristic of a montmorillonite clay containing $\mathrm{Na}^{+}$. The membranes were characterized by contact angle (flat membrane), scanning electron microscopy (SEM) and flow measurements. By means of the contact angle measurements it was seen that the presence of clay favored an increase the hydrophilicity of the membranes. At the hollow fiber membranes, it was observed by SEM that the presence of clay modified the membrane morphology, changing the uniformity of the "fingers" and the macropores and modifying the dense layer in the center of the porous support. The addition of the acid in the internal liquid modified even more the morphology, decreasing the dense layer at the center of the membrane porous support and also changing the "fingers" and the macropores, having a greater uniformity 
of the same with respect to the membrane without the presence of DMF in the internal liquid. From the flow measurements it was possible to observe that the membrane containing 5\% CLNa with the presence of the solvent in the internal liquid obtained a greater flow over time, this occurred due to the presented more uniform morphology of the membrane.

Keywords: polyethersulfone, membrane, hollow fiber, flow measurements.

\section{INTRODUÇÃO}

A tecnologia de membranas foi considerada uma abordagem alternativa e atraente para a separação porque $o$ processo é mais rápido, eficiente em termos de não envolver qualquer alteração de fase. Desde 1960, quando a primeira membrana comercial foi obtida através do método de inversão de fases, houve resultados significativos no desenvolvimento de novas tecnologias de separação por membranas produzidas pela comunidade científica e também no âmbito comercial [1-3]. Após isso, importantes processos de separação por membranas, como osmose inversa, microfiltração, ultrafiltração e separação de gás, foram estabelecidos em larga escala. Atualmente, a aplicação de ultrafiltração na tecnologia de membranas está crescendo muito rapidamente em produtos farmacêuticos, químicos e nas indústrias de papel, papel, semicondutores e lácteas. O principal objetivo da tecnologia de membranas é controlar a estrutura das mesmas, o que afeta o seu desempenho. Assim, pesquisas têm sido conduzidas na tentativa de melhorar o desempenho das membranas [4-7].

Existe uma série de técnicas de preparação de filmes poliméricos porosos para uso em membranas, como a sinterização, estiramento, gravação ("track-etching") e inversão de fases [5,8]. A técnica comum para a preparação de membranas assimétricas de ultrafiltração é a inversão de fases. A produção dessas membranas é bastante influenciada por muitos fatores que incluem os três componentes, que são polímero, solvente e não solvente. A presença de aditivos desempenha um papel crucial no ajuste das propriedades da membrana, que geralmente criam uma estrutura que atribui à membrana uma prevenção maior em relação a formação de macrozavios e melhora a formação e interconectividade dos poros, aumentando assim a hidrofílicidade da membrana [9-11].

Com o intuito de obter membranas com geometria que proporcione uma maior eficiência para as mais variadas aplicações aliada a uma morfologia adequada, é que começaram a se fabricar membranas de fibra oca. Essas são fabricadas geralmente utilizando o processo conhecido como fiação, onde este é uma variação do processo de inversão de fase, no qual o polímero é dissolvido e em seguida extrudado (extrusora com dois orifícios circulares concêntricos) em direção a um banho contendo não solvente, onde vai ocorrer a precipitação [12]. Quando uma solução de polímero viscoso passa através do canal dentro de uma fieira, ele será submetido a diferentes tensões, que podem influenciar os efeitos de orientação da cadeia e do empacotamento, afetando a formação da fibra, morfologia e desempenho de separação [13,14]. Do ponto de vista da aplicação, a membrana de fibra oca apresenta características onde as mesmas vêm sendo aplicadas em diversos setores, tanto industriais como na medicina $[15,16]$.

Os polímeros utilizados para preparação de membranas na forma de fibras são principalmente polisulfonas, polietersulfona (PES), polieterimida, poliimida, poliacrilonitrila e poliésteres. A PES é amplamente utilizada por suas boas características físicas, químicas, estabilidade à hidrolise, propriedades mecânicas e uma ampla faixa de $\mathrm{pH}$ de 1 a 13 para uso. Além disso, a PES pode ser utilizada em temperaturas elevadas, com resistência à oxidação, com isso se tornou um dos principais materiais utilizados na fabricação de membranas [16-18]. Porém, quando comparado com outros polímeros apresenta como principal desvantagem a alta tendência de formar incrustações. Devido a algumas propriedades indesejáveis da PES surge a necessidade de adicionar um agente hidrofílico como aditivo, para a melhoria dessas limitações. Uma alternativa é a produção de híbridos a partir de polímeros com argila [19,20].

Medeiros [21] produziu membranas a partir de nanocompósitos de polietersulfona e argila na proporção de 3 e $5 \%$ em peso, pela técnica de inversão de fases. Como solvente foi utilizado o $N, N$ Dimetilformamida (DMF) e para as membranas de fibra oca foi utilizado também a Polivinilpirrolidona (PVP) como aditivo. A partir dos resultados de FRX, FTIR e DRX das argilas, observou-se que a modificação orgânica da argila Brasgel PA foi eficiente e que a Cloisite 20A é comercialmente organofílica. Pelo DRX das membranas, em geral, elas apresentaram estrutura esfoliada e/ou parcialmente esfoliada. Entretanto, as membranas com as argilas Cloisite $\mathrm{Na} 5 \%$ e a Cloisite 20A (3 e 5\%) evidenciaram estrutura intercalada e/ou parcialmente intercalada. Por análises de MEV, verificou-se que as membranas apresentaram estrutura anisotrópica, com a presença de macroporos no suporte poroso, cuja quantidade aumentou com a inclusão de argila. A partir dos resultados de ângulo de contato com água destilada, notou-se que a inclusão da argila diminuiu o ângulo, ou seja, aumentou a molhabilidade da membrana, sendo esta mais destacada para a argila MMT. Resultado semelhante foi observado para o ângulo de contato com o óleo. Por medida de 
permeabilidade à água destilada, verificou-se que a inclusão e o tipo de argila influenciaram diretamente nas propriedades das membranas, aumentando a hidrofilicidade e favorecendo uma maior permeabilidade, principalmente para a membrana com MMT. Para os resultados com a emulsão oleosa, todas as membranas apresentaram rendimento de separação água/óleo maior que $80 \%$, o que está de acordo com os padrões exigidos pela legislação ambiental. Para as membranas de fibra oca, tanto a introdução da argila quanto a do PVP aumentaram a viscosidade da solução. Por MEV, pôde-se verificar que tanto o tipo quanto a quantidade de argila, e o GAP afetam a morfologia dos poros destas membranas.

Carvalho [22] obteve membranas de poliétersulfona (PES) e poliétersulfona/argila pela técnica de inversão de fase e avaliar a presença de argila na obtenção de membranas para o tratamento de efluentes líquidos. O solvente utilizado foi a dimetilformamida (DMF) e argilas utilizadas foram Brasgel PA (MMT) e Cloisite $\mathrm{Na}(\mathrm{CL} \mathrm{Na})$ nas proporções de 3 a 5\% (em peso). Por difração de raios-X (DRX), as membranas com $3 \%$ das argilas MMT e CL Na aparentemente apresentaram estruturas parcialmente esfoliadas; para a composição com 5\% de CL Na observou-se um pequeno pico, que indica que esta é, possivelmente, uma estrutura intercalada ou microcompósito. A partir dos resultados de microscopia eletrônica de varredura (MEV), visualizou-se que a superfície da membrana de PES puro apresentou uma estrutura aparentemente sem poros, no aumento utilizado e, uma superfície sem rugosidade quando comparada com às membranas com argila. As medidas de ângulo de contato indicaram que a inclusão da argila alterou a capacidade de molhamento das membranas. O fluxo com água destilada para todas as membranas iniciou alto e ao longo do tempo chegou a um patamar de estabilização. Assim, pôde-se concluir que a presença e o teor de argila alteraram a morfologia da membrana, contribuindo para um aumento do fluxo de água.

Arthanareeswaran e Starov [23] estudaram o efeito do solvente na obtenção de membranas de PES por inversão de fases. Eles avaliaram o fluxo de água pura, resistência, estabilidade mecânica e desempenho de separação destas membranas. Os pequisadores utilizaram o $N, N$-dimetilformamida (DMF), N- metil-2pirrolidona (NMP) e dimetil sulfóxido (DMSO) como solventes e avaliaram o desempenho das membranas obtidas. A partir das análises de MEV, eles verificaram que as membranas de PES formaram uma estrutura assimétrica. O número de poros formados sobre a camada superior das membranas de PES, foi resultado do efeito combinado das propriedades termodinâmicas do sistema e da cinética de formação da membrana. A formação da camada macroporosa dessas membranas foi controlada pela taxa de difusão do solvente e do não-solvente (água pura). A ordem do fluxo de água pura das membranas com diferentes solventes foi a seguinte DMF>NMP>DMSO. A resistência da membrana e a estabilidade mecânica diminuíram com a diminuição da concentração do PES, enquanto que aumentaram com a concentração de solventes.

Sendo assim, este estudo tem como objetivo controlar a morfologia das membranas de fibra oca de polietersulfona a partir da introdução do solvente (DMF) no líquido interno para retardar a precipitação e obter poros menores e mais regulares no suporte poroso, e pela adição da argila na solução para atuar como agente porogênico ao longo da seção transversal da membrana híbrida.

\section{MATERIAIS E MÉTODOS}

\subsection{Materiais}

Argila comercial Cloisite $\mathrm{Na}$ (nomeada de CLNa), com CTC (capacidade de troca de cátions) $=90$ meq/100g, fornecida pela Bentonit União Nordeste (BUN). A matriz polimérica utilizada foi a polietersulfona (PES), com nome comercial Veradel ® 3000P, adquirida pela Solvay, na forma de pó de coloração branca.

Para obtenção das membranas foi utilizado o solvente N.N-Dimetilformamida P.A./ACS (DMF), o mesmo também foi utilizado no líquido interno para a produção das membranas, o viscosificante poli(vinilpirrolidona) (PVP), foi utilizado a fim de controlar a viscosidade da solução polimérica, ambos foram produzidos pela Labsynth Produtos para Laboratório Ltda.

\subsection{Preparação das amostras}

A polietersulfona foi seca em estufa a $80{ }^{\circ} \mathrm{C}$ por 24 horas. Para a composição do polímero puro, $70 \%$ em peso mássico do solvente (DMF) foi misturado com $23 \%$ em peso mássico do PES e $7 \%$ em peso mássico do aditivo viscosificante (PVP), sob agitação por 1 hora, a uma velocidade de $1200 \mathrm{rpm}$. Para a composição que contém argila, a solução inicialmente foi preparada contendo $5 \%$ em peso mássico de argila (CL Na) e 70\% em peso mássico do DMF, ficando sob agitação por 1 hora a $1200 \mathrm{rpm}$. Após esse tempo, foi adicionado (23\% PES + 7\% PVP) em peso mássico na solução ficando por igual período sob a mesma agitação. Depois do preparo da solução, a mesma ficou em repouso por um período de 24 horas, para eliminação de bolhas. Os fluxogramas presentes nas Figuras 1 e 2 ilustram os passos da preparação da solução. 


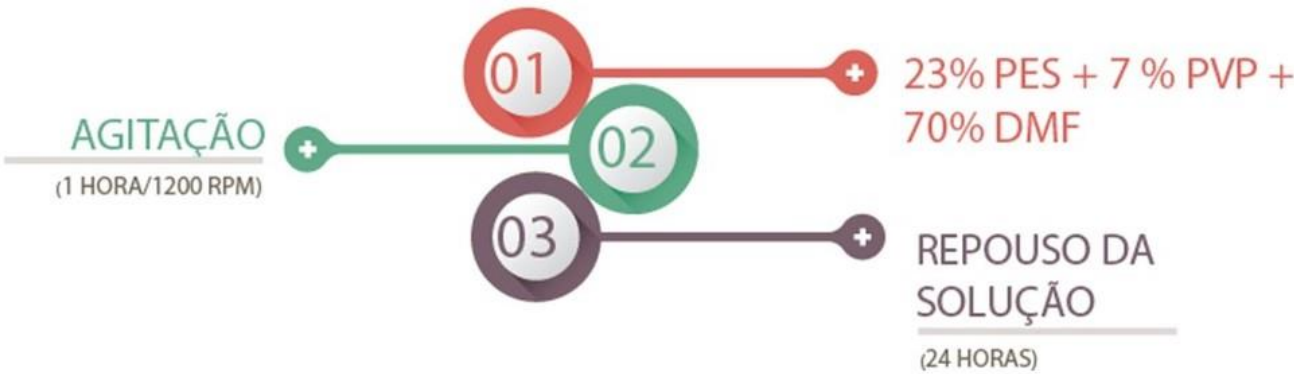

Figura 1: Fluxograma da preparação da solução de PES, contendo (23\% PES + 7\% PVP + 70\% DMF) em peso mássico que permaneceu sob agitação $(1 \mathrm{~h} / 1200 \mathrm{rpm})$ e posterior repouso $(24 \mathrm{~h})$ para a obtenção da membrana de PES.

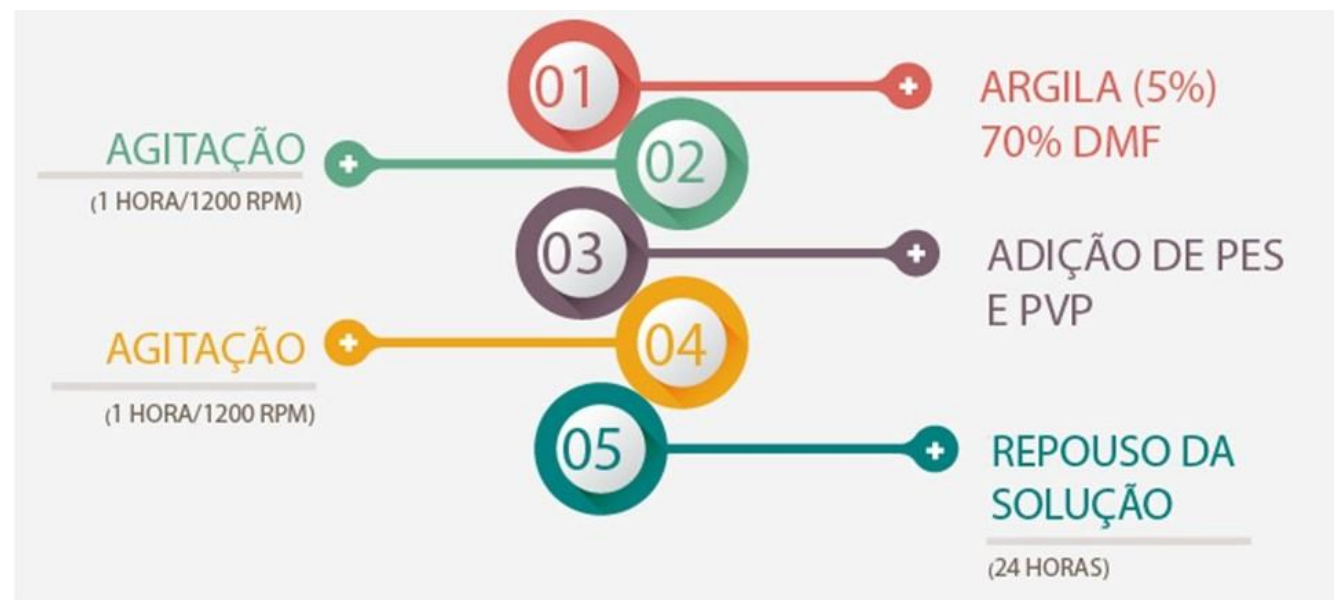

Figura 2: Fluxograma da preparação da solução híbrida, contendo $(5 \%$ argila $+70 \% \mathrm{DMF})$ em peso mássico que permaneceu sob agitação $(1 \mathrm{~h} / 1200 \mathrm{rpm})$, para adição de (23\% PES + 7\% PVP) em peso mássico para agitação (1 h/1200 rpm) e posterior repouso ( 24 h) para a obtenção da membrana híbrida.

Para a obtenção das membranas do tipo fibra oca foram utilizados em torno de $400 \mathrm{~g}$ de solução para que ter-se um fluxo contínuo durante o procedimento. Elas foram preparadas utilizando uma extrusora contendo dois orifícios concêntricos, permitindo a extrusão simultânea do líquido interno e da solução polimérica (Figura 3). Após a extrusão, a solução polimérica entrou em contato com o banho de não-solvente (água destilada), onde a mesma permaneceu até completar a precipitação. Logo após, as fibras foram recolhidas e colocadas em água por $24 \mathrm{~h}$. Com o intuito de modificar a morfologia da membrana e retardar a precipitação dos poros, foi adicionado ao líquido interno $10 \%$ do solvente DMF.

Antes da obtenção das membranas na forma de fibra oca, foram obtidas membranas planas de cada solução preparada, para a realização de algumas caracterizações que só são possíveis a partir desse formato de membrana. Para a obtenção das membranas planas, as soluções anteriormente preparadas foram espalhadas, através de bastões de vidro com espaçamento de aproximadamente 0,2 $\mathrm{mm}$, em placas de vidro e, posteriormente, foram submetidas a um banho de não solvente, no caso água destilada, na temperatura ambiente, de forma que as placas ficassem completamente submersas. As membranas permaneceram no banho até que sua precipitação fosse concluída. Logo após, as mesmas foram removidas das placas, lavadas com água destilada e armazenadas para caracterizações específicas. A Figura 4 ilustra o processo de obtenção das membranas de fibra oca. 

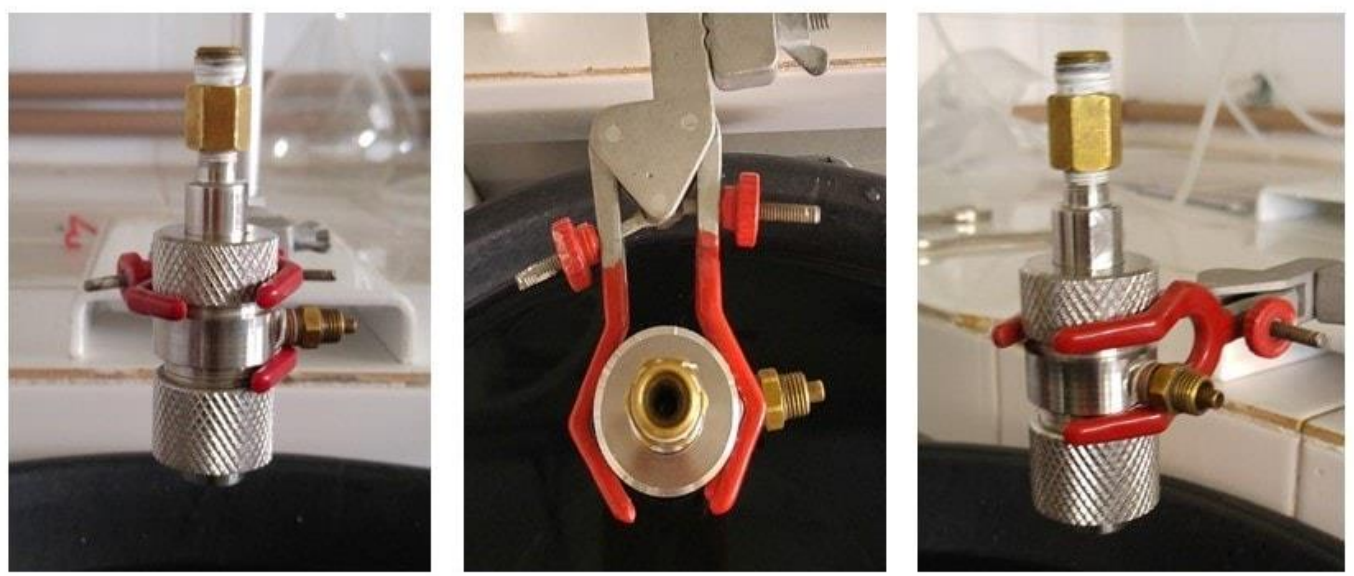

Figura 3: Extrusora utilizada para preparação das membranas.

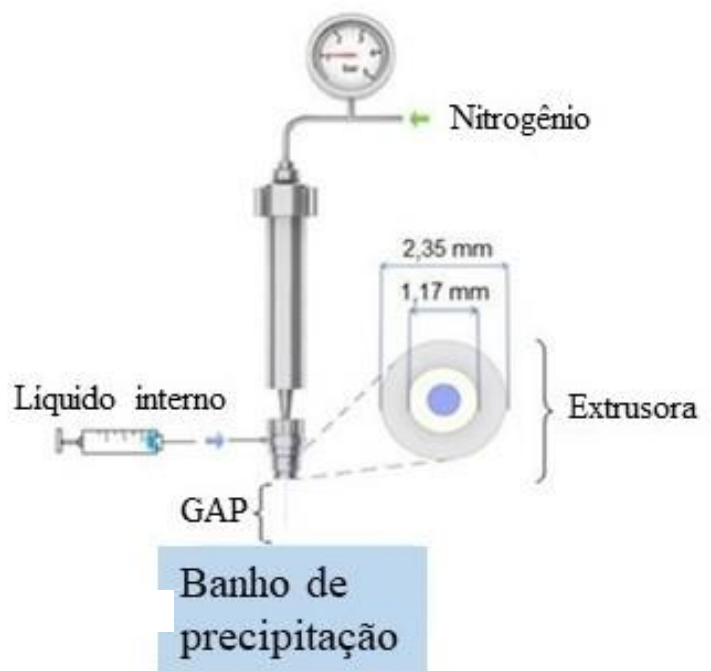

Figura 4: Representação esquemática do processo de obtenção das membranas de fibra oca. (Adaptado de LUITEN et al. [24]).

Para a obtenção das membranas, foram utilizadas as composições, apresentadas na Tabela 1.

Tabela 1: Composições das membranas de fibra oca.

\begin{tabular}{c|c|c|c|c}
\hline Amostras & Solvente (\%) & Polímero (\%) & Argila (\%) & Aditivo (\%) \\
\hline PES Puro & 70 & 23 & 0 & 7 \\
\hline PES + CLNa 5\% & 70 & 21,85 & 1,15 & 7 \\
\hline
\end{tabular}

Os parâmetros para obtenção das membranas de fibra oca foram definidos através de testes realizados em laboratório, variando a viscosidade da solução, o GAP (distância entre a extrusora e o banho de precipitação), o fluxo do líquido interno e também o fluxo da solução polimérica. O experimento realizado foi sob a temperatura ambiente. Esses parâmetros mencionados (Tabela 2) foram controlados a fim de encontrar as melhores condições possíveis para a fiação e obtenção das membranas polimérica e híbrida. 
Tabela 2: Parâmetros de fiação para obtenção da fibra oca.

\begin{tabular}{l|l}
\hline PARÁMETROS & VALORES \\
\hline Viscosidade da solução & $\approx 4200 \mathrm{mPa} . \mathrm{s}$ \\
\hline Temperatura da solução & $\approx 26^{\circ} \mathrm{C}$ \\
\hline GAP & $5 \mathrm{~cm}$ \\
\hline Fluxo do líquido interno & $3 \mathrm{~g} / \mathrm{min}$ \\
\hline Fluxo da solução & $6 \mathrm{~g} / \mathrm{min}$ \\
\hline
\end{tabular}

\subsection{Caracterizações dos materiais}

\subsubsection{Fluorescência de Raios-X (FRX)}

A análise química da argila Cloisite NA, foi realizada pelo método semi quantitativo, em um equipamento EDX 720 da Shimadzu, sob atmosfera de nitrogênio. O material fornecido foi quarteado e prensado em prensa manual em forma de pastilha, com diâmetro de cerca de $15 \mathrm{~mm}$.

\subsubsection{Difração de Raios-X (DRX)}

A análise de DRX, para as argilas, foram conduzidas em um aparelho XRD-6000 Shimadzu, utilizando-se radiação $\mathrm{K} \alpha$ do cobre, tensão de $40 \mathrm{kV}$, corrente de $30 \mathrm{~mA}$, varredura entre 2 a $60^{\circ}$ e velocidade de varredura de $2 \%$ min

\subsection{3 Ângulo de contato}

A análise do ângulo de contato para determinar a hidrofilicidade das membranas foi realizada pelo método da gota séssil, através de um ângulo de contato portátil, modelo Phoenix-i da Suface Eletro Optics - SEO. A gota foi formada manualmente por meio de um dosador micrométrico, a imagem da gota é captada pela câmera embutida no equipamento, onde posteriormente será analisada no software. Está análise foi realizada a partir da membrana plana, tendo em vista que não é possível a realização desta análise com a membrana de fibra oca. Foi realizada uma análise a partir de 30 fotos, utilizando um intervalo de 10 segundos. A análise foi realizada na membrana plana, visto que o formato da fibra oca não é compatível com o teste.

\subsubsection{Microscopia eletrônica de varredura (MEV)}

Para análise da morfologia das membranas de fibra oca, foram realizadas fotomicrografias da seção transversal (ST), o detalhe da seção transversal próximo à superfície externa (STE) e interna (STI). Para análise da seção transversal, as amostras foram fraturadas em nitrogênio líquido para evitar deformação plástica. As superfícies das amostras foram revestidas com ouro com o objetivo de evitar o acúmulo de carga negativa. As análises foram realizadas no equipamento VEGA 3 - TESCAN, operando-se em 30kV.

\subsubsection{Teste de medidas de fluxo}

Para as medidas de fluxo das membranas de fibra oca foram produzidos módulos, a partir de uma pipeta de $20 \mathrm{~cm}$ contendo 5 membranas, em seguida foram impermeabilizadas para a realização da análise com água destilada. Foram utilizadas as pressões de 1,0;1,5 e 2,0 bar, realizando em triplicata para cada composição. $\mathrm{O}$ tempo do teste tem cerca de 50 minutos, dependendo do comportamento do fluxo da membrana.

\section{RESULTADOS E DISCUSSÃO}

\subsection{Fluorescência de raios (FRX)}

A análise química semiquantitativa realizada por FRX para a argila Cloisite Na (CLNa), encontra-se na Tabela 3. As percentagens estão em peso dos óxidos, normalizados a 100\%.

É possível observar que a argila contém os elementos característicos da argila bentonítica, como a alumina $\left(\mathrm{Al}_{2} \mathrm{O}_{3}\right)$ e a sílica $\left(\mathrm{SiO}_{2}\right)$. É visto que se tem a presença de cátions trocáveis em forma de óxidos, como o $\mathrm{MgO}, \mathrm{Na}_{2} \mathrm{O}$ e $\mathrm{CaO}$, devido a argila ser policatiônica. A Cloisite $\mathrm{Na}$ apresenta uma característica naturalmente sódica, devido a presença do $\mathrm{Na}_{2} \mathrm{O}$. É possível afirmar que a argila apresenta um bom grau de pureza, levando em consideração um percentual não tão elevando de $\mathrm{SiO}_{2}$. 
A análise química também permite verificar a presença de minerais acessórios, e elementos característicos da argila em estudo, representados, pelos óxidos de ferro $\left(\mathrm{Fe}_{2} \mathrm{O}_{3}\right)$, cálcio $(\mathrm{CaO})$, titânio $\left(\mathrm{TiO}_{2}\right)$ e outros, como foi observado por Santos [25].

Tabela 3: Análise química por fluorescência de raios-x da argila Cloisite Na.

\begin{tabular}{c|c}
\hline $\begin{array}{l}\text { ELEMENTOS } \\
\text { PRESENTES }\end{array}$ & \multicolumn{2}{l}{} \\
\hline $\mathrm{SiO}_{2}$ & 55,07 \\
\hline $\mathrm{Al}_{2} \mathrm{O}_{3}$ & 27,12 \\
\hline $\mathrm{Fe}_{2} \mathrm{O}_{3}$ & 2,63 \\
\hline $\mathrm{MgO}$ & 4,17 \\
\hline $\mathrm{Na}_{2} \mathrm{O}$ & 10,10 \\
\hline $\mathrm{CaO}$ & 0,35 \\
\hline $\mathrm{TiO}_{2}$ & 0,09 \\
\hline $\mathrm{K}_{2} \mathrm{O}$ & - \\
\hline $\mathrm{SO}_{3}$ & 0,44 \\
\hline $\mathrm{Outros}$ & 0,03 \\
\hline
\end{tabular}

\subsection{Difração de Raios-X}

Na Figura 5 está presente o espectro de difração de raios-X para a argila Cloisite Na. Observa-se que a argila sódica apresentou picos característicos de bentonitas, e uma banda em 5-7 ${ }^{\circ}$ indicando a distância interplanar basal, $\mathrm{d}_{001}$, de $14,82 \AA$, que é característica de montmorilonitas contendo íons $\mathrm{Na}^{+}$na estrutura com uma pequena hidratação [25]. No difratograma é possível visualizar que a argila Cloisite $\mathrm{Na}$ apresenta quase nenhum pico característico de quartzo, indicando assim que a argila está livre de impurezas, como já era esperado, devido ao seu processo de purificação quando da sua produção.

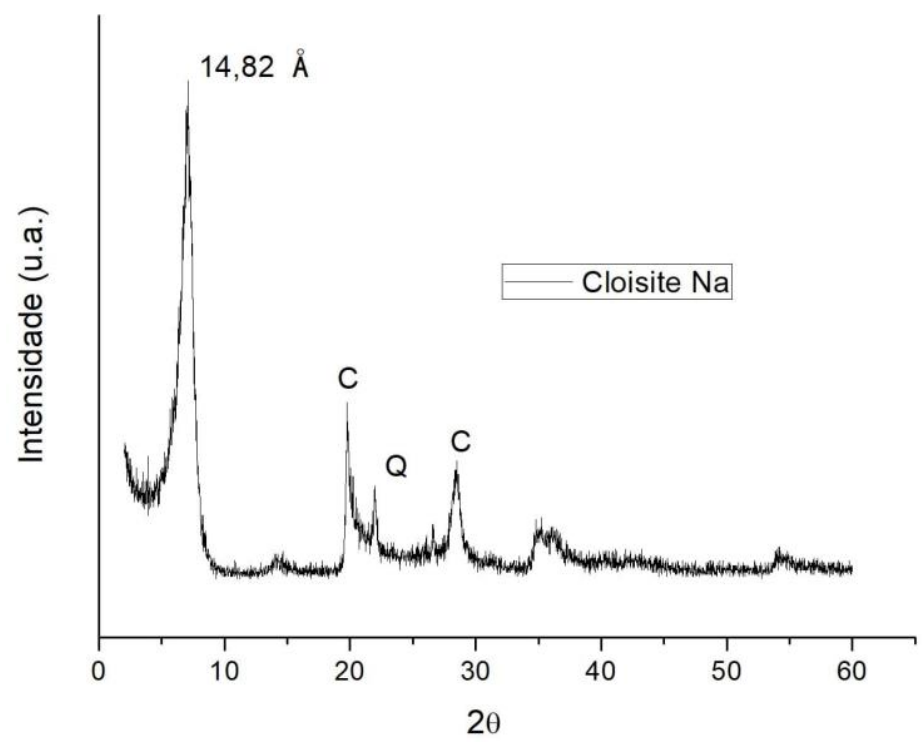

Figura 5: Difratograma de DRX da argila Cloisite Na.

\section{3 Ângulo de contato}

A Figura 6 ilustra a análise de ângulo de contato realizado para as membranas planas produzidas. Para a membrana produzida a partir da PES pura, foi possível observar uma baixa hidrofilicidade, absorvendo uma pequena quantidade de água ao longo do tempo, provavelmente devido à formação de uma camada densa existente na sua superfície.

Já para a membrana com argila aconteceu o que era esperado, visto que houve uma maior diminuição do ângulo inicial e absorção de água em relação à membrana de PES, evidenciando assim que a presença da 
argila atuou como agente porogênico, aumentando a hidrofilicidade da membrana. Ahmad et al. [26] observaram que a adição de diferentes quantidades de $\mathrm{ZnO}$ na membrana de PES aumentou a característica hidrofílica membrana.

A membrana contendo a argila Cloisite Na teve uma diminuição de cerca de $22 \%$ em relação ao ângulo inicial. $\mathrm{O}$ aumento da hidrofilicidade foi maior devido a presença da argila na matriz polimérica para a formação da membrana, determinado isto pela característica hidrofílica da argila Cloisite Na. A presença de um pequeno percentual de materiais acessórios e um maior grau de pureza da argila (Tabela 3), também favoreceu esse comportamento da membrana. Assim, foi possível verificar que a membrana de PES é menos hidrofílica que a membrana contendo argila, devido a uma menor diminuição de $14 \%$ entre o ângulo inicial e final, observado também por Rahimi et al. [27].

A medida do ângulo de contato é uma forma de avaliar a propriedade da hidrofilicidade e hidrofobicidade da superfície da membrana, onde isto implica a energia de interação entre a superfície e o líquido utilizado [27, 28]. Sendo assim de extrema importância no que diz respeito a influência nas propriedades de fluxo e capacidade de anti-incrustação das membranas.

Para todas as membranas produzidas, o ângulo de contato diminuiu em relação ao tempo, provavelmente devido à porosidade das mesmas, fazendo com que a gota preenchesse os poros da superfície da membrana, causando assim uma diminuição do ângulo de contato.

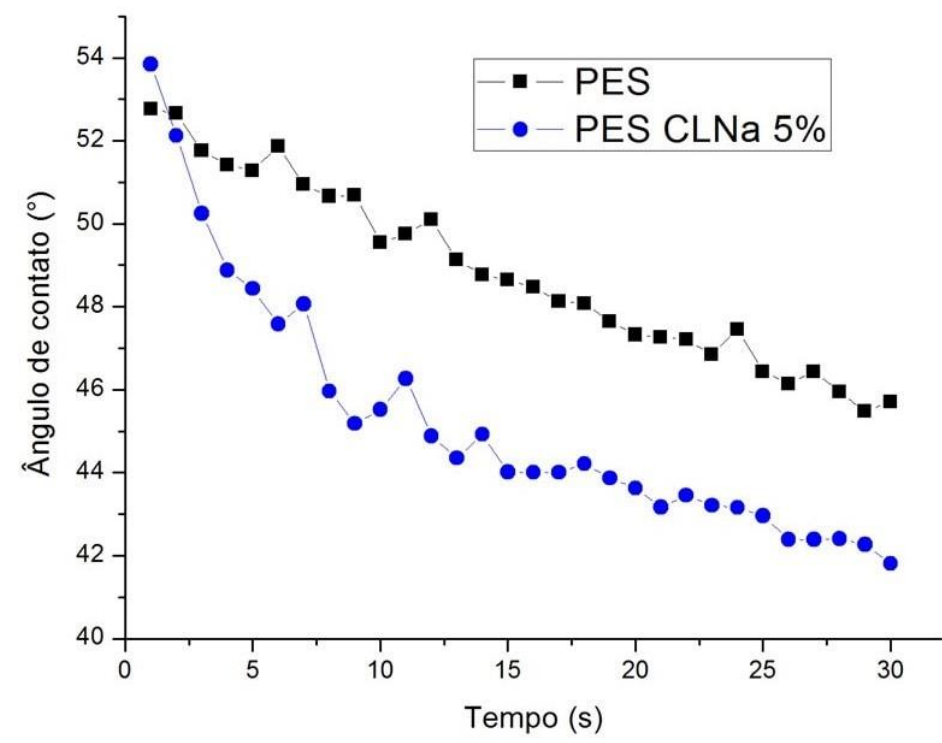

Figura 6: Ângulo de contato para as membranas planas de PES e PES/CLNa.

\subsection{Microscopia eletrônica de varredura (MEV)}

As Figuras 7 a 10 apresentam as fotomicrografias obtidas por MEV da seção transversal das membranas.

É possível observar que todas as membranas apresentam uma estrutura tipicamente assimétrica com a presença de poros e macroporos em toda seção transversal delas. Há uma camada superior extremamente fina e aparentemente densa, onde são sustentados pelo suporte poroso da membrana, com a presença de poros e macroporos e também com a presença de "fingers", sendo eles relacionados à precipitação com atraso da membrana quando a mesma entra em contato com o banho de não-solvente. Os fingers são formados quando a membrana entra em contato com a água, onde ocorre uma decomposição espinodal com tensões localizadas, além disso, essa estrutura também depende da viscosidade da membrana [29, 30]. Houve o aparecimento de uma camada densa entre a camada interna e a camada externa da membrana, podendo ser relacionado com a frente de precipitação da membrana. A camada interna obtida é aparentemente fina e semelhante à camada externa. Essa estrutura foi também observada por Sun et al. [16] e Salahi et al. [31]. Em todas as figuras, três partes foram especificadas como: 1 - camada interna da membrana; 2 - centro do suporte poroso e 3 - camada externa da membrana.

A Figura 7 apresenta as fotomicrografias da seção transversal da membrana de PES pura, para um aumento de 60x. É possível observar a presença de macroporos e também a presença de "fingers" em todo o suporte poroso da membrana. A membrana obteve a camada externa e interna extremamente fina e densa e uma camada densa entre a parte superior e o centro da membrana. 

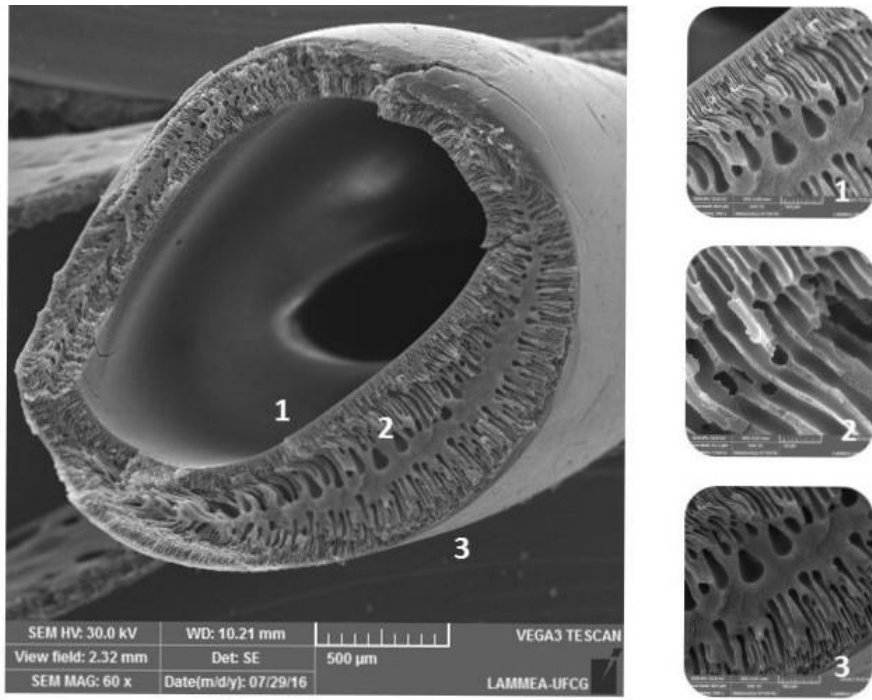

Figura 7: Fotomicrografias de MEV da PES pura.

A Figura 8 ilustra as fotomicrografias da seção transversal da membrana com 5\% de CLNa. É possível observar que a argila Cloisite modificou a estrutura da membrana, comparando com a membrana de PES. Ficou evidente uma maior uniformidade e distribuição dos poros e como também a diminuição da camada densa no centro do suporte poroso da membrana. Houve também uma maior regularidade no tamanho do "fingers", trazendo uma característica morfológica mais uniforme do que a membrana de PES. A introdução de argila para a produção da membrana tem como objetivo não só modificar a morfologia, mas também influenciar diretamente na hidrofilicidade da mesma, como foi observado na análise do ângulo de contato para as membranas planas produzidas com o mesmo percentual de argila das fibras ocas.
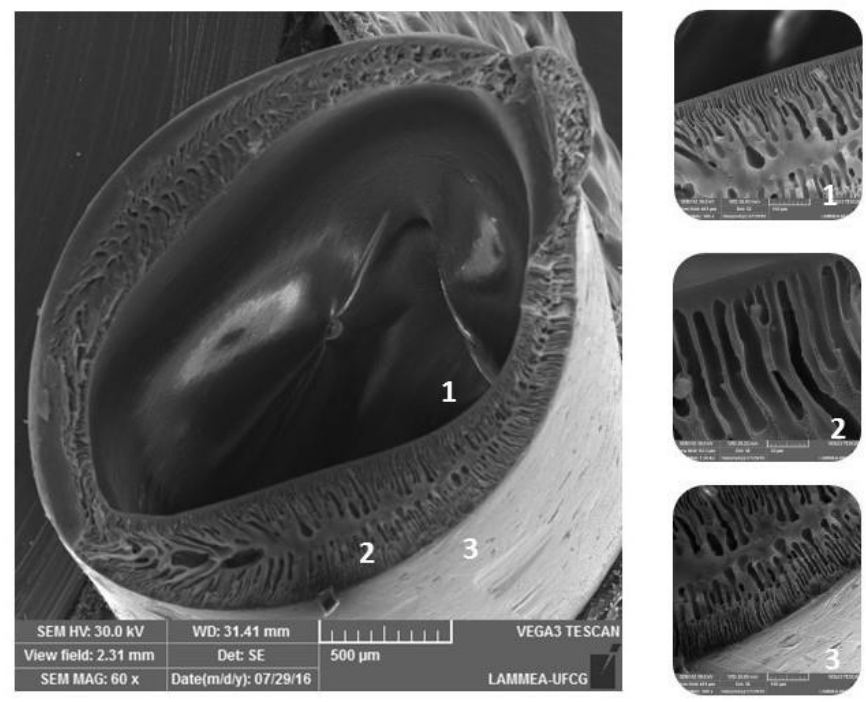

Figura 8: Fotomicrografias de MEV da PES CLNa 5\%.

A Figura 9 ilustra as fotomicrografias da seção transversal da membrana de PES pura com a presença do DMF no líquido interno. É visível a presença de macroporos, em uma quantidade baixa e bem distribuídos em todo o suporte poroso. Os "fingers" obtiveram uma boa uniformidade, tanto no tamanho como na forma. Já a camada densa no centro do suporte poroso teve sua espessura fina, mostrando que o solvente atuou na frente de precipitação da membrana quando comparado com a membrana de PES sem a presença do solvente no líquido interno. 

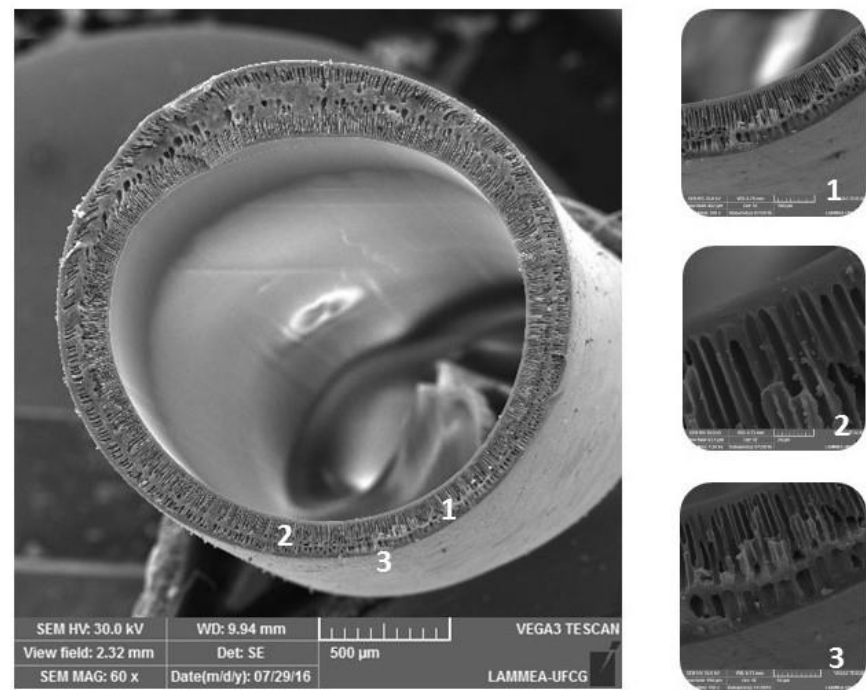

Figura 9: Fotomicrografias de MEV da PES pura (10\% solvente).

A Figuras 10 apresenta as fotomicrografias de MEV da seção transversal da membrana com 5\% de CLNa. Foi possível observar uma diminuição na quantidade e tamanho dos macroporos no suporte poroso, e também uma maior uniformidade dos "fingers". A camada densa no centro do suporte poroso quase desapareceu, mostrando uma forte influência do DMF no líquido interno. Assim, foi possível ver que o solvente alterou diretamente a morfologia das membranas com MMT, proporcionando uma melhoria na uniformidade do suporte poroso e a redução da camada densa no centro do suporte.
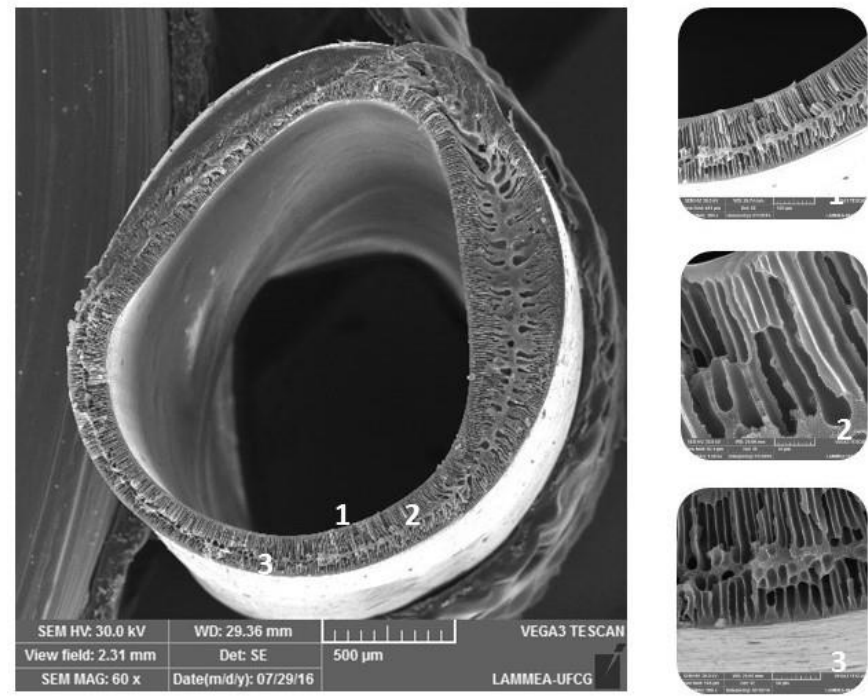

Figura 10: Fotomicrografias de MEV da PES CLNa 5\% (10\% solvente).

Salimi et al. [32] obtiveram morfologia semelhante, onde a membrana de PES possui uma estrutura assimétrica composta por uma camada superior seguida por uma camada porosa com a presença dos "fingers". Devido à boa miscibilidade da NMP (1-metil-2-pirrolidona) com água, o mesmo pôde migrar da solução e passar para a água do banho de coagulação. Ao mesmo tempo, as moléculas de água não são capazes de difundir na solução, isto devido às fracas interações do polímero com a água. Assim, a solução começa a solidificar formando a camada superior da membrana. A difusão das moléculas de água nas partes internas da membrana inicia a formação de núcleos e o NMP favorece o crescimento dos poros. Consequentemente, a membrana de fibra oca de PES forma uma morfologia no suporte poroso do formato de "fingers".

A partir das fotomicrografias apresentadas foi possível verificar que a argila e o DMF no líquido interno tiveram influência diretamente na morfologia das mesmas. A introdução do DMF no líquido interno teve uma forte influência nos macroporos presentes no suporte poroso, pois além de estarem em menor 
quantidade, diminuíram também o seu tamanho e ainda tiveram uma maior uniformidade, como pode ser visto no comparativo entre as figuras 5 e 7 . Os "fingers" apresentaram uma maior linearidade em todo o suporte poroso. A camada densa no centro do suporte poroso teve sua espessura reduzida devido a presença da argila e também do DMF no líquido interno.

\subsection{Medidas de fluxo}

A Figura 11 apresenta os resultados do ensaio de medida de fluxo com água destilada com o intuito de avaliar o fluxo de água através da membrana, sob distintas pressões (1,0; 1,5 e 2,0 bar).

No geral, é possível observar que para todas as composições o fluxo teve a tendência de aumentar com o passar do tempo, esse comportamento só não foi observado para a membrana PES CLNa 5\% (Figura 6), esse comportamento também foi observado por Xiang et al. [33]. Para todas as membranas foi visto que o permeado começou a ser coletado após 20 minutos do início do ensaio, isto ocorrendo em função do preenchimento dos poros da membrana pela água, onde a mesma vai tendo os seus poros preenchidos até que se comece a ser coletado o permeado.

A membrana que apresentou um melhor padrão nas 3 pressões estudadas foi a PES CLNa $5 \%$ com o DMF no líquido interno, mostrando o que já era esperando pelo o que foi visto do teste do ângulo de contato. A membrana de PES com a presença do DMF também apresentou um bom fluxo no intervalo de tempo estudado. Assim, esse comportamento pode ter ocorrido devido à presença do solvente (DMF) no líquido interno para produção da membrana, alterando a hidrofilicidade da membrana e dando a mesma um maior fluxo do que as outras membranas estudadas. Ficou visível que a adição da argila também aumento o fluxo em relação a membrana de PES pura, isto pode ser proveniente da característica hidrofílica da argila, onde também foi observado este comportamento no ângulo de contato.
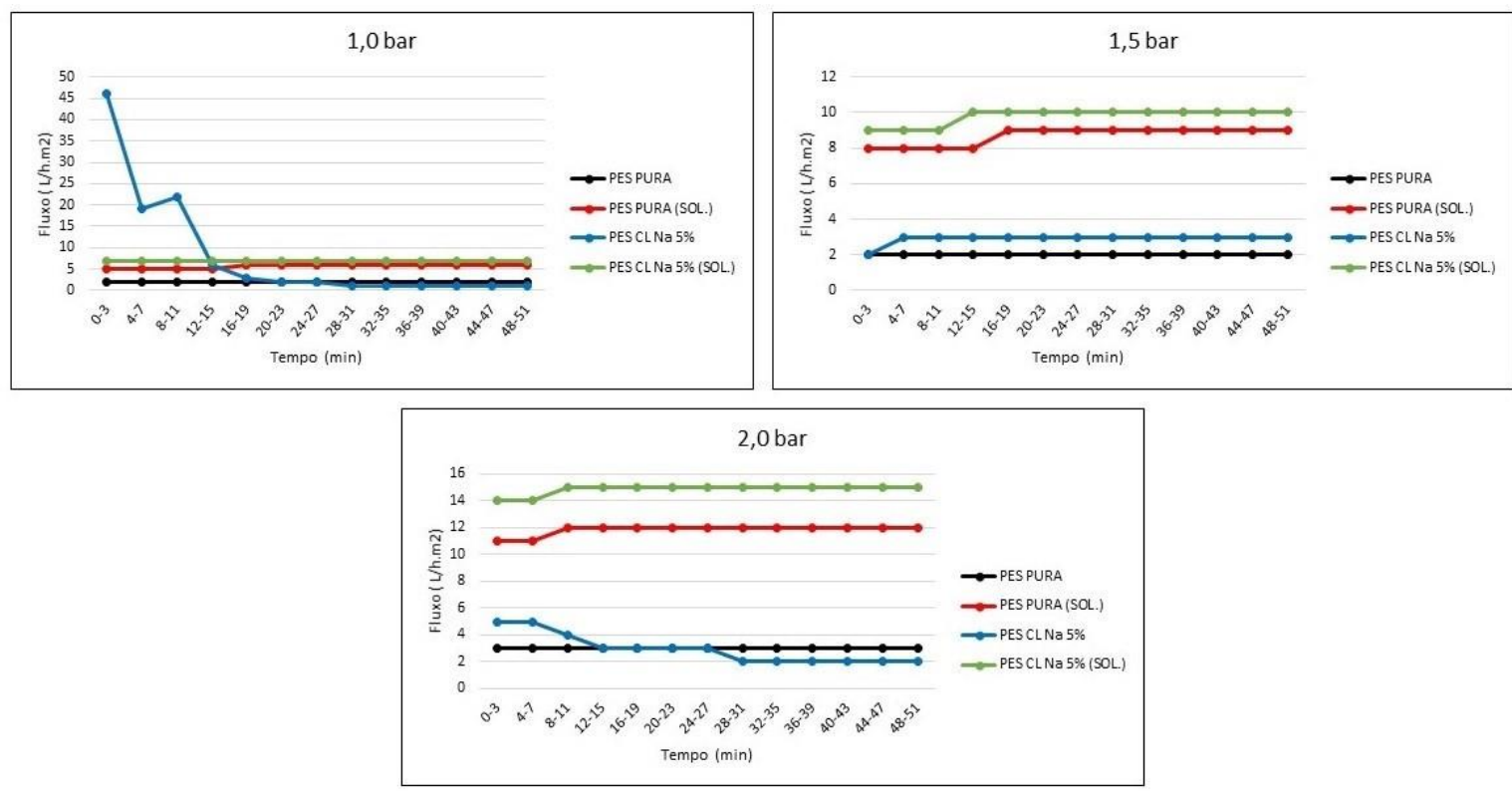

Figura 11: Fluxo de água destilada em função do tempo para as membranas de PES e PES/argila.

Na Figura 12 está apresentado o fluxo estabilizado para todas as membranas em função das três pressões estudadas. É visível que a membrana com 5\% CLNa (10\% solvente) obteve um fluxo maior para todas as pressões, onde é possível fazer a ligação do ângulo de contato em que essa composição apresentou melhores resultados de hidrofilicidade. A membrana de PES CLNa 5\% (10\% solvente) obteve um fluxo estabilizado de 7, 10 e $15 \mathrm{~L} / \mathrm{h} . \mathrm{m}^{2}$ para as pressões de 1,0; 1,5 e 2,0 bar, respectivamente. Um fluxo mais elevado para esta composição pode também ser atribuído ao caráter hidrofílico da argila Cloisite Na, trazendo assim um maior fluxo para a membrana como também a morfologia apresentada pela membrana.

Para composição contendo argila $5 \%$ de CL Na o efeito foi contrário ao que foi visto para a membrana com $5 \%$ CLNa (10\% solvente). Este efeito pode ser avaliado, devido à interação entre polímero/argila/solvente e como também a morfologia da membrana, e a quantidade e tamanho dos poros e a espessura da pele filtrante. Assim, influenciando diretamente na permeabilidade, criando um caminho tortuoso adequando no interior da membrana, obtendo um baixo fluxo. 


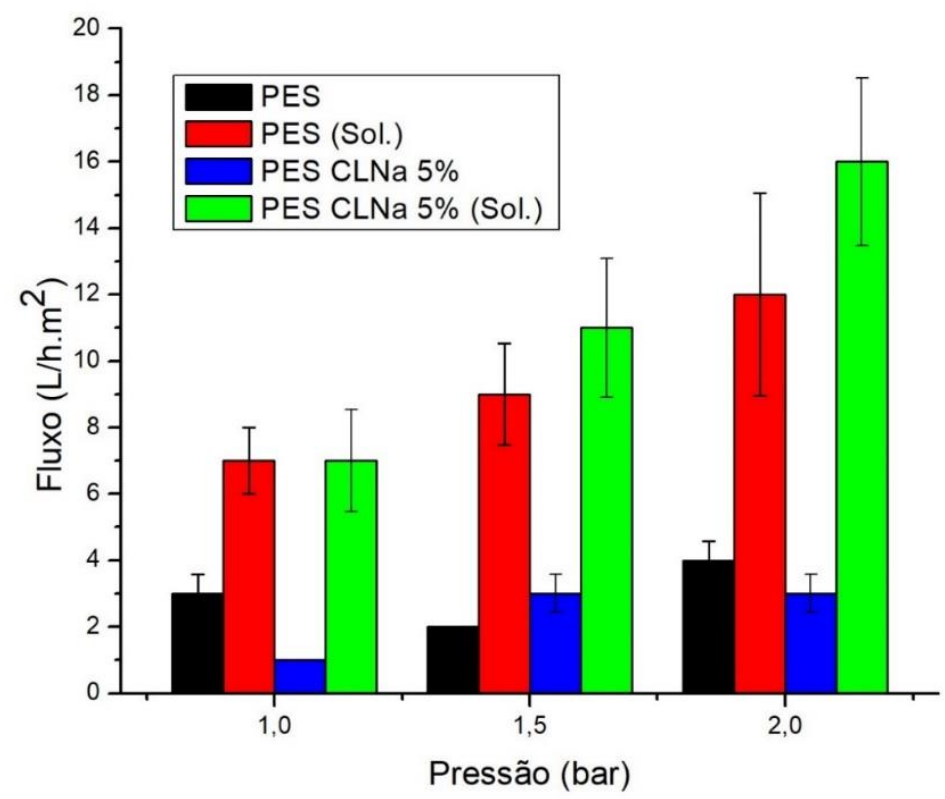

Figura 12: Fluxo estabilizado de todas as membranas a pressão de 1,0; 1,5 e 2,0 bar no tempo de 50 minutos.

\section{CONCLUSÕES}

Membranas de fibra oca de PES e PES/argila foram produzidas com sucesso. Por FRX ficou visível que a argila Cloisite $\mathrm{Na}$ apresenta uma característica naturalmente sódica, tendo a mesma um considerável grau de pureza devido ao percentual de $\mathrm{SiO}_{2}$. A partir do DRX ficou evidente que a argila pertence ao grupo das montmorilonitas, apresentando um elevado grau de pureza. Foi possível observar que a introdução da argila influenciou diretamente na análise do ângulo de contato para as membranas, mostrando que o pequeno percentual de argila modificou a hidrofilicidade da mesma. A presença do solvente no líquido interno para a produção da membrana, juntamente com a argila, modificaram a morfologia da membrana, tendo a mesma fingers bem definidos, com um suporte poroso com poros e macroporos e uma camada densa no centro do suporte mais fina. Essa modificação da morfologia para as membranas produzidas com o DMF no líquido interno favoreceu um melhor fluxo durante um período de 50 minutos, mostrando a influência da argila e do solvente.

\section{AGRADECIMENTOS}

Os autores agradecem ao Labmat (Laboratório de Engenharia de Materiais/CCT/UFCG) pelos experimentos realizados, ao MCTI/CNPq, CAPES/PNPD e à CAPES, pelo apoio financeiro.

\section{BIBLIOGRAFIA}

[1] BAKER, R. W., Membrane technology and applications, 2 ed., John Wiley \& Sons Inc, California,USA, 2004.

[2] ANADÃO, P., Ciência e tecnologia de membranas, Artliber Editora Ltda. São Paulo, 2010.

[3] HABERT, A. C., BORGES, C. P., NÓBREGA, R., Processo de separação com membranas, $1^{\mathrm{a}}$ ed., EPapers Serviços Editoriais Ltda, Rio de Janeiro, 2006.

[4] IDRIS, A., ZAIN, N. M., NOORDIN, M. Y. "Synthesis, characterization and performance of asymmetric polyethersulfone (PES) ultrafiltration membranes with polyethylene glycol of different molecular weights as additives", Desalination, v. 207, pp. 324-339, Março. 2007.

[5] WANG, X. L., QIAN, H. J., CHEN, L. J., et al., "Dissipative particle dynamics simulation on the polymer membrane formation by immersion precipitation", Journal of Membrane Science, v. 311, n. 1, pp. 251-258, Março. 2008.

[6] LIU, F., HASHIM, N. A., LIU, Y., et al., "Progress in the production and modification of PVDF membranes", Journal of Membrane Science, v. 375, n. 1, pp. 1-27, Jun. 2011. 
[7] SILVA, M. C., LIRA, D. S., FREITAS, L. F., et al., "Membranas cerâmicas assimétricas tubulares: influência do tempo de deposição da camada filtrante na morfologia das membranas", Matéria, v. 20, n. 2, pp. 335-343, Jun. 2015.

[8] BAKERI, G. H., ISMAIL, A. F., DASHTARZHANDI, M. R., et al., "Porous PES and PEI hollow fiber membranes in a gas-liquid contacting Process - A comparative study", Journal of Membrane Science, v. 475, pp. 57-6, Jul. 2015.

[9] BARTH, C., GONÇALVES, M. C., PIRES, A. T. N., et al., "Asymmetric polysulfone and polyethersulfone membranes: effects of thermodynamic conditions during formation on their performance". Journal of Membrane Science, v. 169, pp. 287-299, Maio. 2000.

[10] WU, H., TANG, B., WU, P., “Optimizing polyamide thin film composite membrane covalently bonded with modified mesoporous silica nanoparticles”, Journal of Membrane Science, v. 428, n. 1, pp. 341-348, Fev. 2013

[11] XU, J., FENG, X., GAO, C., "Surface modification of thin-film-composite polyamide membranes for improved reverse osmosis performance", Journal of Membrane Science, v. 370, n. 1-2, pp. 116-123, Março. 2011.

[12] SENGUR, R., LANNOY, C. F., TURKEN, T., et al., "Fabrication and characterization of hydroxylated and carboxylated multiwalled carbon nanotube/polyethersulfone (PES) nanocomposite hollow fiber membranes". Desalination, v. 359, pp. 123-140, Março. 2015.

[13] HILAL, N., ISMAIL, A. F., WRIGHT, C. J., Membrane Fabrication, CRC Press, Taylor and Fracis Group, New York, 2015.

[14] WANG, K. Y., MATSUURA, T., CHUNG, T. S., et al., "The effects of flow angle and shear rate within the spinneret on the separation performance of poly(ethersulfone) (PES) ultrafiltration hollow fiber membranes", Journal of Membrane Science, v. 240, pp. 67-79, Set. 2004.

[15] CHUNG, T. S., QIN, J. J., GU, J., "Effect of shear rate within the spinneret on morphology, separation performance and mechanical properties of ultrafiltration polyethersulfone hollow fiber membranes", Chemical Engineering Science, v. 55, pp. 1077-1091, Março. 2000.

[16] SUN, D., YANG, Q. C., SUN, H. L., et al., "Effects of PES support layer structure on pervaporation performances of PDMS/PES hollow fiber composite membranes", Desalination and Water Treatment, v. 57, n. 20, pp. 9123-9135, Março. 2016.

[17] DANG, J, ZHANG, Y., DU, Z., et al., "Antibacterial properties of PES/CuCl(2) three-bore hollow fiber UF membrane”. Water Science \& Technology, v. 66, p. 799-803, Jul. 2012.

[18] YA, L., ROU-XI, C., FU-JUAN, L., "Comparison between electrospun and Bubbfil-spun Polyether sulfone fibers", Matéria, v. 19, n. 4, p. 363-369, Out/Dez. 2014.

[19] MANSOURPANAH, Y., MADAENI, S.S., RAHIMPOUR, A., et al., "Fabrication new PES-based mixed matrix nanocomposite membranes using polycaprolactone modified carbon nanotubes as the additive: property changes and morphological studies", Desalination, v. 277, n. 1, pp. 171-177, Ago. 2011.

[20] ZHU, L.J., ZHU, L.P., JIANG, J.H., et al., "Hydrophilic and anti-fouling polyethersulfone ultrafiltration membranes with poly(2-hydroxyethyl methacrylate) grafted silica nanoparticles as additive". Journal of Membrane Science, v.451, pp.157-168, Fev. 2014.

[21] MEDEIROS, V.N., "Desenvolvimento de membranas de poliétersulfona por inversão de fases", Tese de D.Sc., CCT/UFCG, Campina Grande, PB, Brasil, 2016.

[22] CARVAlHO, T. C., MEDEIROS, V. N., LEITE, A. M. D. L., et al., "Membranas de poliétersulfona/argila e sua permeabilidade à água", Matéria, v. 22, n. 2, pp. 335-343, Jun. 2017.

[23] ARTHANAREESWARAN, G., STAROV, V. M., "Effect of solvents on performance of polyethersulfone ultrafiltration membranes: Investigation of metal ion separations", Desalination, v.267, pp. 57-63, Fev. 2011.

[24] LUITEN, M. W. J., RAAIJMAKERS, M. J. T., WINNUBST, L., et al., "Towards a generic method for inorganic porous hollow fibers preparation with shrinkage-controlled small radial dimensions, applied to Al2O3, Ni, SiC, stainless teel, and YSZ”, Journal of Membrane Science, v. 407-408, pp. 155-163, Jul. 2012.

[25] SANTOS, P. S., Ciência e Tecnologia de Argilas. $2^{a}$ Edição Revisada e Ampliada, Edgar Blücher, São Paulo, v. 1, 1989.

[26] AHMAD, A. L., ABDulKRIM, A. A., SHAFIE, Z. M. H. M., et al., "Fouling evaluation of PES/ZnO mixed matrix hollow fiber membrane”, Desalination, v. 403, pp. 53-63, Fev. 2017. 
[27] RAHIMI, M., ZINADINI, S., ZINATIZADEH, A. A., et al., "Hydrophilic goethite nanoparticle as a novel antifouling agent in fabrication of nanocomposite polyethersulfone membrane", Journal of Applied Polymer Science, v. 133, n. 26, Jul. 2016.

[28] ZINADINI, S., ZINATIZADEH, A. A., RAHIMI, M., et al., "Preparation of a novel antifouling mixed matrix PES membrane by embedding graphene oxide nanoplates". Journal of Membrane Science, v. 453, pp. 292-301, Março. 2014.

[29] QIN, J. J., CHUNG, T. S., "Effects of orientation relaxation and bore fluid chemistry on morphology and performance of polyethersulfone hollow fibers for gas separation". Journal of Membrane Science, v. 229, pp. 1-9, Fev. 2004.

[30] XU, Z. L., QUSAY, F. A., "Polyethersulfone (PES) hollow fiber ultrafiltration membranes prepared by PES/non-solvent/NMP solution”. Journal of Membrane Science, v. 233, n. 1, pp. 101-111, Abril. 2004.

[31] SALAHI, A., MOHAMMADI, T., BEHBAHANI, R. M., et al., "Asymmetric polyethersulfone ultrafiltration membranes for oily wastewater treatment: synthesis, characterization, ANFIS modeling, and performance". Journal of Environmental Chemical Engineering, v. 3, n. 1, pp. 170-178, Março 2015.

[32] SALIMI, E., GHAEE, A., ISMAIL, A. F., "Performance and antifouling enhancement of polyethersulfone hollow fiber membranes incorporated with highly hydrophilic hydroxyapatite nanoparticles". Royal Society of Chemistry Advances, v. 6, n. 50, pp. 44480-44488, Abril. 2016.

[33] XIANG, T., TANG, M., LIU, Y., et al., "Preparation and characterization of modified polyethersulfone hollow fiber membranes by blending poly (styrene-alt-maleic anhydride)", Desalination, v. 295, pp. 26-34, Jun. 2012.

\section{ORCID}

Rodholfo da Silva Barbosa Ferreira Sandriely Sonaly Lima de Oliveira Edcleide Maria Araújo Keila Machado Medeiros Hélio de Lucena Lira Amanda Melissa Damião Leite
https://orcid.org/0000-0002-5547-994X https://orcid.org/0000-0002-7430-0597 https://orcid.org/0000-0003-4906-864X https://orcid.org/ 0000-0001-9250-1432 https://orcid.org/0000-0002-1527-9935 https://orcid.org/0000-0003-1597-4230 